\title{
Special issue on artificial intelligence/machine learning in travel
}

\section{B. Vinod ${ }^{1}$}

Published online: 13 March 2021

(c) The Author(s), under exclusive licence to Springer Nature Limited 2021
Over the past decade, Artificial Intelligence has proved invaluable in a range of industry verticals such as automotive and assembly, life sciences, retail, oil and gas, and travel. The leading sectors adopting AI rapidly are Financial Services, Automotive and Assembly, High Tech and Telecommunications. Travel has been slow in adoption, but the opportunity for generating incremental value for AI over other analytics is extremely high (Chui et al. 2018).

In September 2019, Ian Yeoman and I discussed creating a special issue for the Journal of Revenue and Pricing Management on Artificial Intelligence in Travel. Information from airlines and vendors on $\mathrm{AI}$ in travel has been sporadic, usually discussed at industry conferences. Yet it was abundantly clear to me based on my interactions with travel suppliers, software vendors, OTAs and GDSs that they were leveraging core concepts in Artificial Intelligence and Machine Learning to create new value propositions or improve on existing applications related to travel. This was an opportunity to showcase in a single issue the breadth and scope of what individuals in these organizations were focused on with applications and business process.

The research papers...

An excellent contribution from Rodrigo Acuna-Agost, Eoin Thomas and Alix Lh'eritier from Amadeus, who propose a new method to estimate price elasticity for deep learning-based choice models with an excellent set of references. The insights they provide are particularly relevant for airline offers based on customer segment and context.

Ahmed Abdelghany and Ching-Wen Huang from EmbryRiddle University and Khaled Abdelghany from Southern Methodist University propose a novel reinforcement learning approach to calibrate itinerary choice models and measure schedule profitability.

Melvin Woodley from Sabre solves the attribution problem of associating sales or revenue to individual marketing

B. Vinod

benvinod@yahoo.com

1 Southlake, USA efforts. Companies invest broadly across different platforms such as Google or Facebook, and it is important to understand the marginal impact of a specific marketing program or initiative on revenue. He uses a novel approach by casting the well-known Koyck distributed lag model in state space form to analyze the effectiveness of each marketing channel and subsequent allocation of marketing budgets.

The paper by Ravi Kumar, Wei Wang, Ahmed Simrin, Sivarama Krishnan Arunachalam and Bhaskar Rao Guntreddy and Darius Walczak on competitive revenue management models is a collaboration between PROS and Etihad Airways. Their paper proposes a demand model that captures realistic competitive dynamics by considering two types of customer behaviors: airline's loyal customers who prefer to buy from the airline even if their price is not the lowest in the market and fully flexible customers who buy the lowest fare in the market. They develop a Bayesian machine learningbased demand forecasting methodology for these models in both class-based and class-free settings that explicitly considers competitive market information.

Norbert Remenyi and Xiaodong Luo from Sabre discuss practical limitations of the choice-based demand models found in the literature to estimate demand from sales transaction data. They propose modifications and extensions under partial availability and extend the Expected Maximization (EM) algorithm for nonhomogeneous product sets. The data preprocessing and solution techniques are useful for practitioners.

The practice papers...

The paper on recommender systems by Amine Dadoun, Michael Defoin Platel, Thomas Fiig, Corinne Landra and Raphael Troncy from Amadeus highlight the central role of recommender systems to create personalized offers and its growing importance with IATA's New Distribution Capability messaging standard. It is a well-researched paper and truly relevant to the future of airline retailing.

Michael Byrd from Yum! and Ross Darrow from Charter and Go make the case for contextual bandits, a reinforcement learning technique for personalizing offers in retailing. They provide insights into the use of Thompson sampling, a 
popular exploration heuristic and how they can be deployed. They discuss the step improvement that can be achieved with contextual bandits, despite greater computational complexity incurred when contextual features are included in the model.

Tomasz Szymanski from Nordea Bank and Ross Darrow from Charter and Go discuss the important topic of shelf placement on agency storefronts. While airlines focus on offer creation, the GDS desktop must display non-homogenous content that is addressed in this paper. A shelf product assortment method is proposed for categorizing airline offers into utility levels, thus facilitating the itinerary selection process for travelers.

Jian Wang from Realpage outlines a practical application of reinforcement learning used to determine reference rents for apartments. He demonstrates how the new approach outperforms the traditional rules-based approach.

Shriguru Nayak, Nitin Gautam and Sergey Shebalov from Sabre apply machine learning models to estimate market size and market share from competitive future schedules and augmented data sources, a key component for developing airline schedules. They also discuss how revenue management practices can be improved with access to data from network planning.

The paper by Rimo Das, Harshinder Chaddha and Somnath Banerjee from LodgIQ focuses on forecasting market demand considering seasonality and market events. They examine a variety of machine learning techniques that the data were calibrated upon and report on the accuracy of the forecasts.

In January 2018, an AI initiative was established at Sabre to identify industry-relevant problems suitable for AI-based solutions, raise internal awareness and accelerate adoption. This initiative also led to the creation and distribution of an internal AI newsletter, quarterly town halls to monitor progress and discuss use cases that I was responsible for. My contribution to the special issue reflects this initiative and steps taken to solve a range of problems in travel.
Foremost on the minds of corporations as they leverage AI for competitive advantage is how to scale AI across the organization. Deborah Leff and Kenneth Lim from IBM draw upon their extensive experience working with many companies to provide insights into the various organizational barriers to scale AI, the importance of executive sponsorship and recommend best practices. This paper is a "must read" for anyone who is a practitioner of AI.

The futures article...

Ross Darrow's future's article is thought provoking. "The Future of AI is the Market" paints a picture of how the future travel distribution landscape will be influenced by interactions in the marketplace and less on targeted one-off solutions.

I would like to take this opportunity to thank all the anonymous referees I reached out to over the past few months to provide feedback on the submitted papers. This special issue would not have been possible without your feedback and requests for revisions.

\section{Reference}

Chui, M., R. Chung, N. Henke, S. Malhotra, J. Manyika, M. Miremadi, and P. Nel. 2018. Notes from the AI Frontier: Applications and Value of Deep Learning. McKinsey.com, April 2018.

Publisher's Note Springer Nature remains neutral with regard to jurisdictional claims in published maps and institutional affiliations.

B. Vinod serves as Chief Scientist and Senior Vice President at Sabre (2008-2020). Before rejoining Sabre in 2004, he was Vice President at Sabre Airline Solutions, responsible for Pricing and Yield Management. 